Journal of Applied AnALysis

Vol. 13, No. 1 (2007), pp. 133-149

\title{
ON MINIMAX INEQUALITIES IN TOPOLOGICAL SPACES WITHOUT CONVEXITY STRUCTURE
}

\author{
H.-S. LU \\ Received March 22, 2005 and, in revised form, October 12, 2005
}

\begin{abstract}
In this paper, we give a new nonempty intersection theorem in general topological spaces without convexity structure. As its applications, some new minimax inequalities are obtained in general topological spaces without convexity structure.
\end{abstract}

\section{INTRODUCTION AND PRELIMINARIES}

In 1998, Zhang and Ma [16] established the following nonempty intersection theorem in topological vector spaces and gave its applications to minimax inequalities.

Theorem A. Let $E$ and $F$ be Hausdorff topological vector spaces, let $X \subset$ $E, Y \subset F$ be nonempty convex subsets, and let $A$ be a subset of $X \times Y$ such that

(i) for each $x \in X$, the set $\{y \in Y:(x, y) \notin A\}$ is convex or empty;

2000 Mathematics Subject Classification. Primary: 47H04, 49J35, 52A07.

Key words and phrases. Intersection theorem, minimax inequality, transfer compactly closed-valued (open-valued), acyclic set, set-valued mapping.

This work is supported by Natural Science Foundation of Education Committee of Jiangsu Province.

ISSN 1425-6908 C Heldermann Verlag. 
(ii) for each $y \in Y$, there exists a closed subset $X_{y} \subset X$ such that the set $\{x \in X:(x, y) \in A\} \subset X_{y}$.

Suppose that there exists a subset $B$ of $A$ and a compact convex subset $K$ of $X$ such that $B$ is closed in $X \times Y$ and

(iii) for each $y \in Y$, the set $\{x \in K:(x, y) \in B\}$ is nonempty and convex. Then

$$
\bigcap_{y \in Y} X_{y} \cap K \neq \emptyset
$$

Recently, Lu and Zhang [10] proved the following nonempty intersection theorem in $H$-spaces, which generalizes Theorem A.

Theorem B. Let $X$ be a Hausdorff topological space, $(X,\{\Gamma(A)\})$ be an $H$ space, $C: Y \rightarrow 2^{X}$ be a set-valued mapping with closed values, and let $M$, $N$ be two subsets of $X \times Y$ with $M \subset N$. Suppose the following conditions are fulfilled:

(i) for each $y \in Y$, the set $\{x \in X:(x, y) \in N\} \subset C(y)$;

(ii) for each $x \in X$, the set $\{y \in Y:(x, y) \notin M\}$ is $H$-convex or empty.

Suppose also that there exists a subset $P$ of $M$ and a compact subset $K$ of $X$ such that $P$ is closed in $X \times Y$, and

(iii) for each $y \in Y$, the set $\{x \in K:(x, y) \in P\}$ is nonempty acyclic.

Then

$$
\bigcap_{y \in Y} C(y) \cap K \neq \emptyset \text {. }
$$

In this paper, our purpose is to establish a new nonempty intersection theorem in general topological spaces without convexity structure, and next as its applications, we give some new minimax inequalities in general topological spaces without convexity structure. Our results generalize and improve many recent known results, see for example [5]-[8] and [10], [14], [16], [17].

Let $X$ be a set. We shall denote by $2^{X}$ the family of all subsets of $X$, by $\langle X\rangle$ the family of nonempty finite subsets of $X$. For any $A \in\langle X\rangle$, let $|A|$ denote the cardinality of $A$. Let $\Delta_{n}$ denote the standard $n$-dimensional simplex with vertices $\left\{e_{0}, \ldots, e_{n}\right\}$, where $e_{i}$ is the $(i+1)$ th unit vector in $\mathbb{R}^{n+1}$. If $\emptyset \neq J \subset\{0, \ldots, n\}$, then we denote by $\Delta_{|J|-1}$ the convex hull of the vertices $\left\{e_{i}: i \in J\right\}$.

We recall the notion of an $H$-space introduced by Bardaro and Ceppitelli [2].

Let $X$ be a topological space, $\{\Gamma(A)\}$ be a family of nonempty contractible subsets of $X$ indexed by $A \in\langle X\rangle$ such that $\Gamma(A) \subset \Gamma\left(A^{\prime}\right)$, whenever $A \subset A^{\prime}$. The pair $(X,\{\Gamma(A)\})$ is called an $H$-space. Given an $H$-space $(X,\{\Gamma(A)\})$, a nonempty subset $D$ of $X$ is called $H$-convex if $\Gamma(A) \subset D$ for each $A \in\langle D\rangle$. 
For a nonempty subset $E$ of $(X,\{\Gamma(A)\})$, we define the $H$-convex hull of $E$, denoted the $H-\operatorname{co} E$ as

$$
H-\operatorname{co} E=\bigcap\{D \subset X: E \subset D \text { and } D \text { is } H \text {-convex }\} \text {. }
$$

Clearly, $H-\operatorname{co} E$ is $H$-convex and is the smallest $H$-convex set containing $E$.

A nonempty topological space $X$ is said to be acyclic if all of its reduced Čech homology groups over rationals vanish. In particular, any nonempty contractible space is acyclic, and thus any nonempty convex or star-shaped set is acyclic. A nonempty subset $D$ of a topological space $X$ is said to be compactly closed (respectively, compactly open) in $X$ if for each nonempty compact subset $C \subset X, D \cap C$ is closed (respectively, open) in $C$. The compact closure and the compact interior of $D$ (see [3]) are defined by

$$
\begin{aligned}
\operatorname{ccl} D & =\bigcap\{G: D \subset G \text { and } G \text { is compactly closed in } X\}, \text { and } \\
\operatorname{cint} D & =\bigcup\{G: G \subset D \text { and } G \text { is compactly open in } X\},
\end{aligned}
$$

respectively. It is easy to see that $\operatorname{ccl} D$ (respectively, $\operatorname{cint} D$ ) is compactly closed (respectively, compactly open) in $X$ and for each nonempty compact subset $C$ of $X$, we have $(\operatorname{ccl} D) \cap C=\operatorname{cl}_{C}(D \cap C)$ and $(\operatorname{cint} D) \cap C=$ $\operatorname{int}_{C}(D \cap C)$, where $\operatorname{cl}_{C}(D \cap C)$ and int $C(D \cap C)$ denote the closure and the interior of $D \cap C$ in $C$, respectively.

Definition 1.1 (see [3]). Let $X$ be a nonempty set, $Y$ be a topological space, $F: X \rightarrow 2^{Y}$ a set-valued mapping. $F$ is called transfer compactly closed-valued (respectively, transfer compactly open-valued) if for each $x \in$ $X$ and for each nonempty compact subset $C$ of $Y, y \notin F(x) \cap C$ (respectively, $y \in F(x) \cap C)$ implies that there exists an $x^{\prime}$ such that $y \notin \operatorname{cl}_{C}\left(F\left(x^{\prime}\right) \cap C\right)$ (respectively, $y \in \operatorname{int}_{C}\left(F\left(x^{\prime}\right) \cap C\right)$ ).

Remark 1.1. Each closed-valued (respectively, open-valued) set-valued mapping $F: X \rightarrow 2^{Y}$ is transfer closed-valued (respectively, transfer openvalued) (see [15, Definitions 6 and 7]) and is also compactly closed-valued (respectively, compactly open-valued). Each transfer closed-valued (respectively, transfer open-valued) set-valued mapping $F: X \rightarrow 2^{Y}$ is transfer compactly closed-valued (respectively, transfer compactly open-valued) and the inverse in not true in general.

Definition 1.2 (see [3]). Let $X$ be a nonempty set, $Y$ be a topological space, $f: X \times Y \rightarrow \mathbb{R}$ be a function. For some $\lambda \in \mathbb{R}, f(x, y)$ is said to be $\lambda$-transfer compactly lower (respectively, upper) semicontinuous in $y$ if for each compact subset $C$ of $Y$ and for each $y \in C$,

$$
\{x \in X: f(x, y)>\lambda\} \neq \emptyset
$$


(respectively, $\{x \in X: f(x, y)<\lambda\} \neq \emptyset$ ) implies that there exists a relatively open neighborhood $N(y)$ of $y$ in $C$ and an $x^{\prime} \in X$ such that $f\left(x^{\prime}, z\right)>\lambda$ (respectively, $f\left(x^{\prime}, z\right)<\lambda$ ) for all $z \in N(y)$.

Remark 1.2. It is easy to prove that if a set-valued mapping $T: X \rightarrow 2^{Y}$ is defined by

$$
T(x)=\{y \in Y: f(x, y) \leq \lambda\}
$$

(respectively, $T(x)=\{y \in Y: f(x, y) \geq \lambda\}$ ) for some $\lambda \in \mathbb{R}$, then $T$ is transfer compactly closed-valued if and only if $f(x, y)$ is $\lambda$-transfer compactly lower (respectively, upper) semicontinuous in $y$.

Let $X$ and $Y$ be two topological spaces, $T: X \rightarrow 2^{Y}$ be a set-valued mapping. $T$ is said to be upper semicontinuous if for each open subset $G$ of $Y$, the set $\{x \in X: T(x) \subset G\}$ is open in $X$.

Throughout this paper, all topological spaces are assumed to be Hausdorff.

\section{A NONEMPTy INTERSECTION THEOREM}

Our main result is the following Theorem 2.1 which is needed in this paper.

Theorem 2.1. Let $X$ and $Y$ be two topological spaces, $C: Y \rightarrow 2^{X}$ be a set-valued mapping, and let $M, N$ be two subsets of $X \times Y$. Suppose the following conditions are fulfilled:

(i) $C$ is transfer compactly closed-valued;

(ii) for each $y \in Y$, the set $\{x \in X:(x, y) \in N\} \subset C(y)$.

Suppose also that there exists a subset $P$ of $M$ and a compact subset $K$ of $X$ such that $P$ is closed in $X \times Y$, and

(iii) for each $A \in\langle Y\rangle$ with

$$
A \cap\{y \in Y:(x, y) \notin N\} \neq \emptyset
$$

for all $x \in K$, there exists a continuous mapping $\varphi_{A}: \Delta_{|A|-1} \rightarrow Y$ such that

$$
\varphi_{A}\left(\Delta_{|A \cap\{y \in Y:(x, y) \notin N\}|-1}\right) \subset\{y \in Y:(x, y) \notin M\}
$$

for all $x \in K$;

(iv) for each $y \in Y$, the set $\{x \in K:(x, y) \in P\}$ is nonempty acyclic.

Then

$$
\bigcap_{y \in Y} C(y) \cap K \neq \emptyset \text {. }
$$


Proof. We show that the family $\{\operatorname{ccl} C(y) \cap K: y \in Y\}$ has the finite intersection property. Suppose it does not have the finite intersection property. Then there exists $A=\left\{y_{0}, \ldots, y_{n}\right\} \in\langle Y\rangle$ such that

$$
\bigcap_{y \in A} \operatorname{ccl} C(y) \cap K=\emptyset \text {. }
$$

It follows that

$$
K \subset \bigcup\{(X \backslash \operatorname{ccl} C(y)) \cap K: y \in A\} .
$$

Since each $\operatorname{ccl} C(y)$ is compactly closed, we can assume that there exists a non-negative continuous partition of unity $\left\{\beta_{0}, \ldots, \beta_{n}\right\}$ subordinated to the open covering $\{(X \backslash \operatorname{ccl} C(y)) \bigcap K: y \in A\}$, that is, for each $i \in\{0, \ldots, n\}$, $\beta_{i}: K \rightarrow[0,1]$ is continuous such that for each $x \in K, \sum_{i=0}^{n} \beta_{i}(x)=1$ and for each $i \in\{0, \ldots, n\}$,

$$
\beta_{i}(x)=0 \text { for } x \notin\left(X \backslash \operatorname{ccl} C\left(y_{i}\right)\right) \cap K .
$$

In other words, for each $i \in\{0, \ldots, n\}$ and $x \in K, \beta_{i}(x) \neq 0$ implies that

$$
x \in\left(X \backslash \operatorname{ccl} C\left(y_{i}\right)\right) \cap K \subset\left\{x \in X:\left(x, y_{i}\right) \notin N\right\} .
$$

Define a mapping $g: K \rightarrow \Delta_{n}$ by

$$
g(x)=\sum_{i=0}^{n} \beta_{i}(x) e_{i} \text { for all } x \in K .
$$

Then clearly $g$ is continuous. For each $x \in K$, let us define $J(x)=\{i \in$ $\left.\{0, \ldots, n\}: \beta_{i}(x) \neq 0\right\}$ and $B(x)=\left\{y_{i}: i \in J(x)\right\}$, then

$$
\emptyset \neq B(x) \subset A \cap\{y \in Y:(x, y) \notin N\}
$$

by (2.1). Therefore by (iii), there is a continuous mapping $\varphi: \Delta_{n} \rightarrow Y$ such that

$$
\varphi\left(\Delta_{|A \cap\{y \in Y:(x, y) \notin N\}|-1}\right) \subset\{y \in Y:(x, y) \notin M\} \text { for all } x \in K .
$$

Define a continuous mapping $f: K \rightarrow Y$ as follows:

$$
f(x)=\varphi(g(x)) \text { for all } x \in K .
$$

By (2.2),

$$
f(x)=\varphi(g(x)) \in \varphi\left(\Delta_{|B(x)|-1}\right) \subset\{y \in Y:(x, y) \notin M\}
$$

for all $x \in K$. This shows that

$$
(x, f(x)) \notin M \text { for all } x \in K .
$$

On the other hand, we define a set-valued mapping $G: Y \rightarrow 2^{K}$ by

$$
G(y)=\{x \in K:(x, y) \in P\} \text { for all } y \in Y .
$$

By (iv), $G(y)$ is nonempty acyclic for all $y \in Y$. Since $P$ is closed in $X \times Y$, it is easy to see that each $G(y)$ is closed in $K$ and the graph 
of $G$ is closed in $Y \times K$. Hence by Corollary 9 in [1, p. 111], $G$ is an upper semicontinuous set-valued mapping defined on $Y$. Consequently, so is the set-valued mapping $F: \Delta_{n} \rightarrow 2^{K}$ defined by $F(\mu)=G(\varphi(\mu))$ for all $\mu \in \Delta_{n}$. By virtue of Lemma 1 in [12], there exists a point $\bar{\mu} \in \Delta_{n}$ such that $\bar{\mu} \in g(F(\bar{\mu}))=g(G(\varphi(\bar{\mu})))$, and so there is a point $\bar{x} \in G(\varphi(\bar{\mu})) \subset K$ such that $\bar{\mu}=g(\bar{x})$. Let $\bar{y}=\varphi(\bar{\mu})$, then $\bar{y}=\varphi(g(\bar{x}))=f(\bar{x})$ and $\bar{x} \in G(\bar{y})$, i.e.,

$$
(\bar{x}, f(\bar{x}))=(\bar{x}, \bar{y}) \in P \subset M .
$$

This contradicts (2.3). Therefore the family $\{\operatorname{ccl} C(y) \cap K: y \in Y\}$ has the finite intersection property. Since $K$ is compact and each $\operatorname{ccl} C(y)$ is compactly closed, we must have

$$
\bigcap_{y \in Y} \operatorname{ccl} C(y) \cap K \neq \emptyset \text {. }
$$

Now we show that

$$
\bigcap_{y \in Y} C(y) \cap K=\bigcap_{y \in Y} \operatorname{ccl} C(y) \cap K .
$$

Clearly, $\bigcap_{y \in Y} C(y) \bigcap K \subset \bigcap_{y \in Y} \operatorname{ccl} C(y) \bigcap K$. So we only need to show that

$$
\bigcap_{y \in Y} \operatorname{ccl} C(y) \cap K \subset \bigcap_{y \in Y} C(y) \cap K .
$$

Suppose, by the way of contradiction, that there exists an

$$
x \in \bigcap_{y \in Y} \operatorname{ccl} C(y) \cap K=\bigcap_{y \in Y} \operatorname{cl}_{K}(C(y) \cap K)
$$

and a $y \in Y$ such that $x \notin C(y) \cap K$. Since $C$ is transfer compactly closedvalued, there exists a point $y^{\prime} \in Y$ such that $x \notin \mathrm{cl}_{K}\left(C\left(y^{\prime}\right) \cap K\right)$, which is a contradiction. Hence

$$
\bigcap_{y \in Y} C(y) \cap K=\bigcap_{y \in Y} \operatorname{ccl} C(y) \cap K \neq \emptyset .
$$

This completes the proof.

Remark 2.1. Theorem 2.1 generalizes Theorem B in the following ways:

(1) an $H$-space $(X,\{\Gamma(A)\})$ is replaced by a general topological space without convexity structure;

(2) the condition that $C$ is a closed-valued mapping is weakened to the condition that $C$ is a transfer compactly closed-valued mapping;

(3) the assumption of $M \subset N$ in Theorem B is dropped; 
(4) condition (ii) of Theorem B is a special case of condition (iii) of Theorem 2.1. We show that condition (ii) of Theorem B implies condition (iii) of Theorem 2.1. In fact, let $A \in\langle Y\rangle$ with

$$
A \cap\{y \in Y:(x, y) \notin N\} \neq \emptyset
$$

for all $x \in K$. By Theorem 2.1 in [13], there exists a continuous mapping $\varphi_{A}: \Delta_{|A|-1} \rightarrow Y$ such that

$$
\varphi_{A}\left(\Delta_{|B|-1}\right) \subset \Gamma(B) \text { for each } B \in\langle A\rangle .
$$

Since

$$
\emptyset \neq A \cap\{y \in Y:(x, y) \notin N\} \in\langle A\rangle
$$

and

$$
\emptyset \neq A \cap\{y \in Y:(x, y) \notin N\} \in\langle\{y \in Y:(x, y) \notin M\}\rangle
$$

for each $x \in K$, by (2.4) and condition (ii) of Theorem B, we have

$$
\begin{aligned}
& \varphi_{A}\left(\Delta_{|A \cap\{y \in Y:(x, y) \notin N\}|-1}\right) \subset \Gamma(A \cap\{y \in Y:(x, y) \notin N\}) \\
& \subset\{y \in Y:(x, y) \notin M\}
\end{aligned}
$$

for each $x \in K$. Hence condition (iii) of Theorem 2.1 holds.

\section{Minimax inequalities}

Theorem 3.1. Let $X$ and $Y$ be two topological spaces, and let e, $f, g, h: X \times$ $Y \rightarrow \mathbb{R}$ be functions. Let

$$
\beta=\inf _{K \in \bar{K}} \sup _{y \in Y} \min _{x \in K} h(x, y),
$$

where

$$
\bar{K}=\{K \subset X: K \text { is compact acyclic subset of } X\} \text {. }
$$

Suppose the following conditions are fulfilled:

(i) for each $(x, y) \in X \times Y, e(x, y) \leq f(x, y), g(x, y) \leq h(x, y)$;

(ii) for each $t>\beta, e(x, y)$ is t-transfer compactly lower semicontinuous in $x$;

(iii) for each $A \in\langle Y\rangle$ with

$$
A \cap\{y \in Y: f(x, y)>t\} \neq \emptyset
$$

for all $t>\beta, x \in X$, there exists a continuous mapping $\varphi_{A}: \Delta_{|A|-1} \rightarrow$ $Y$ such that

$$
\varphi_{A}\left(\Delta_{|A \cap\{y \in Y: f(x, y)>t\}|-1}\right) \subset\{y \in Y: g(x, y)>t\}
$$

for all $t>\beta, x \in X$; 
(iv) $h(x, y)$ is lower semicontinuous on $X \times Y$ and the set $\{x \in K$ : $h(x, y)<t\}$ is nonempty acyclic or empty for each $t>\beta, K \in \bar{K}$ and $y \in Y$.

Then

$$
\inf _{x \in X} \sup _{y \in Y} e(x, y) \leq \inf _{K \in \bar{K}} \sup _{y \in Y} \min _{x \in K} h(x, y)=\beta .
$$

If $X$ is compact acyclic, then

$$
\inf _{x \in X} \sup _{y \in Y} e(x, y) \leq \sup _{y \in Y} \min _{x \in X} h(x, y) .
$$

Proof. If $\beta=+\infty$, then the theorem is obviously true. Hence we may assume that $\beta<+\infty$. Now let $\lambda>\beta$ be fixed and define a set-valued mapping $C: Y \rightarrow 2^{X}$ as follows:

$$
C(y)=\{x \in X: e(x, y) \leq \lambda\} \text { for all } y \in Y .
$$

Let

$$
\begin{aligned}
& M=\{(x, y) \in X \times Y: g(x, y) \leq \lambda\}, \\
& N=\{(x, y) \in X \times Y: f(x, y) \leq \lambda\}, \quad \text { and } \\
& P=\{(x, y) \in X \times Y: h(x, y) \leq \lambda\} .
\end{aligned}
$$

By (i), for each $y \in Y$,

$$
\{x \in X:(x, y) \in N\} \subset\{x \in X: e(x, y) \leq \lambda\}=C(y) .
$$

By (ii) and Remark 1.2, $C: Y \rightarrow 2^{X}$ is transfer compactly closed-valued. By (iii), we know that for each $A \in\langle Y\rangle$ with

$$
A \cap\{y \in Y:(x, y) \notin N\} \neq \emptyset
$$

for all $x \in X$, there exists a continuous mapping $\varphi_{A}: \Delta_{|A|-1} \rightarrow Y$ such that

$$
\varphi_{A}\left(\Delta_{|A \cap\{y \in Y:(x, y) \notin N\}|-1}\right) \subset\{y \in Y:(x, y) \notin M\}
$$

for each $x \in X$. It is easy to verify that $P$ is closed in $X \times Y$ and $P \subset M$. Let $K$ be a compact acyclic subset of $X$ such that

$$
\lambda>\sup _{y \in Y} \min _{x \in K} h(x, y) .
$$

Then for any $y \in Y$, the set $\{x \in K: h(x, y) \leq \lambda\}$ is nonempty and we know the set

$$
\{x \in K: h(x, y) \leq \lambda\}=\bigcap_{\varepsilon>0}\{x \in K: h(x, y)<\lambda+\varepsilon\}
$$


is acyclic (this follows from the continuity of Čech homology. See Lemma 1.3 in [11]). Thus by Theorem 2.1,

$$
\bigcap_{y \in Y} C(y) \cap K \neq \emptyset
$$

that is, there exists $x_{0} \in K$ such that

$$
e\left(x_{0}, y\right) \leq \lambda \text { for all } y \in Y .
$$

Hence

$$
\inf _{x \in X} \sup _{y \in Y} e(x, y) \leq \lambda .
$$

Since the above inequality holds for any $\lambda>\beta$, we simply let $\lambda$ decrease to $\beta$ to obtain (3.1). This completes the proof.

Remark 3.1. Theorem 3.1 does not require the space $Y$ possess any convexity structure. As a result, Theorem 3.1 generalizes and improves Theorem 3 in [16] and Theorem 2.2 in [10] from topological vector spaces or $H$-spaces with abstract convexity structure to general topological spaces without convexity structure.

Corollary 3.1. Let $X$ and $Y$ be two topological spaces, and let $e, f, g, h: X \times Y \rightarrow \mathbb{R}$ be functions. Let

$$
\beta=\inf _{K \in \bar{K}} \sup _{y \in Y} \min _{x \in K} h(x, y),
$$

where

$$
\bar{K}=\{K \subset X: K \text { is compact acyclic subset of } X\} .
$$

Suppose the following conditions are fulfilled:

(i) for each $(x, y) \in X \times Y, e(x, y) \leq f(x, y) \leq g(x, y) \leq h(x, y)$;

(ii) for each $t>\beta, e(x, y)$ is t-transfer compactly lower semicontinuous in $x$;

(iii) for each $A \in\langle Y\rangle$ with

$$
A \cap\{y \in Y: g(x, y)>t\} \neq \emptyset
$$

for all $t>\beta, x \in X$, there exists a continuous mapping $\varphi_{A}: \Delta_{|A|-1} \rightarrow$ $Y$ such that

$$
\varphi_{A}\left(\Delta_{|A \cap\{y \in Y: g(x, y)>t\}|-1}\right) \subset\{y \in Y: g(x, y)>t\}
$$

for all $t>\beta, x \in X$;

(iv) $h(x, y)$ is lower semicontinuous on $X \times Y$ and the set $\{x \in$ $K: h(x, y)<t\}$ is nonempty acyclic or empty for each $t>\beta, K \in \bar{K}$ and $y \in Y$. 
Then

$$
\inf _{x \in X} \sup _{y \in Y} e(x, y) \leq \inf _{K \in \bar{K}} \sup _{y \in Y} \min _{x \in K} h(x, y)=\beta .
$$

If $X$ is compact acyclic, then

$$
\inf _{x \in X} \sup _{y \in Y} e(x, y) \leq \sup _{y \in Y} \min _{x \in X} h(x, y) .
$$

Taking $e=f=g=h$ in Corollary 3.1, we have the following corollary.

Corollary 3.2. Let $X$ and $Y$ be two topological spaces, $f: X \times Y \rightarrow \mathbb{R}$ be a real-valued function, and let

$$
\beta=\inf _{K \in \bar{K}} \sup _{y \in Y} \min _{x \in K} f(x, y),
$$

where

$$
\bar{K}=\{K \subset X: K \text { is compact acyclic subset of } X\} \text {. }
$$

Suppose the following conditions are fulfilled:

(i) for each $A \in\langle Y\rangle$ with

$$
A \cap\{y \in Y: f(x, y)>t\} \neq \emptyset
$$

for all $t>\beta, x \in X$, there exists a continuous mapping $\varphi_{A}: \Delta_{|A|-1} \rightarrow$ $Y$ such that

$$
\varphi_{A}\left(\Delta_{|A \cap\{y \in Y: f(x, y)>t\}|-1}\right) \subset\{y \in Y: f(x, y)>t\}
$$

for all $t>\beta, x \in X$

(ii) $f(x, y)$ is lower semicontinuous on $X \times Y$ and the set $\{x \in$ $K: f(x, y)<t\}$ is nonempty acyclic or empty for each $t>\beta, K \in \bar{K}$ and $y \in Y$.

Then

$$
\inf _{x \in X} \sup _{y \in Y} f(x, y)=\inf _{K \in \bar{K}} \sup _{y \in Y} \min _{x \in K} f(x, y)=\beta .
$$

If $X$ is compact acyclic, then

$$
\min _{x \in X} \sup _{y \in Y} f(x, y)=\sup _{y \in Y} \min _{x \in X} f(x, y) .
$$

Proof. By Corollary 3.1, we have

$$
\inf _{x \in X} \sup _{y \in Y} f(x, y) \leq \inf _{K \in \bar{K}} \sup _{y \in Y} \min _{x \in K} f(x, y)=\beta .
$$

Since

$$
\inf _{x \in X} \sup _{y \in Y} f(x, y) \geq \inf _{K \in \bar{K}} \sup _{y \in Y} \min _{x \in K} f(x, y)=\beta
$$

is always true, we have

$$
\inf _{x \in X} \sup _{y \in Y} f(x, y)=\inf _{K \in \bar{K}} \sup _{y \in Y} \min _{x \in K} f(x, y)=\beta .
$$


If, in addition, $X$ is compact acyclic, then we have

$$
\min _{x \in X} \sup _{y \in Y} f(x, y)=\sup _{y \in Y} \min _{x \in X} f(x, y) .
$$

This completes the proof.

Remark 3.2. Corollary 3.2 generalizes Theorem 4 in [7] and Corollary 2.3 in $[10]$ in several aspects.

Theorem 3.2. Let $X$ and $Y$ be two topological spaces, $T: X \rightarrow 2^{Y}$ be an upper semicontinuous set-valued mapping with closed acyclic values, $S: X \rightarrow 2^{Y}$ be a set-valued mapping. Let $e, f, g: X \times Y \rightarrow \mathbb{R}$ be functions and

$$
\beta=\sup _{y \in Y} \inf _{x \in X} g(x, y)
$$

Suppose the following conditions are fulfilled:

(i) for each $(x, y) \in X \times Y, f(x, y) \leq g(x, y)$;

(ii) for each $t>\beta, g(x, y)$ is t-transfer compactly upper semicontinuous in $y$;

(iii) for each $A \in\langle X\rangle$ with

$$
A \cap\{x \in X: f(x, y)<t\} \neq \emptyset
$$

for each $t>\beta$ and $y \in Y$, there exists a continuous mapping $\varphi_{A}: \Delta_{|A|-1} \rightarrow X$ such that

$$
\varphi_{A}\left(\Delta_{|A \cap\{x \in X: f(x, y)<t\}|-1}\right) \subset\{x \in X: e(x, y)<t\}
$$

for all $t>\beta$ and $y \in Y$;

(iv) for each $x \in X$,

$$
T(x) \cap S(x) \neq \emptyset \quad \text { and } \quad K=\bigcup_{x \in X} S(x)
$$

is compact acyclic subset of $Y$.

Then

$$
\inf _{x \in X, y \in T x} e(x, y) \leq \sup _{y \in Y} \inf _{x \in X} g(x, y)
$$

Proof. We can assume that the right-hand side of (3.3) is not $+\infty$. If the conclusion of Theorem 3.2 is false, then there exists a real number $t$ such that

$$
\inf _{x \in X, y \in T x} e(x, y)>t>\sup _{y \in Y} \inf _{x \in X} g(x, y) .
$$

For the above $t$, let us define a set-valued mapping $C: X \rightarrow 2^{Y}$ as follows:

$$
C(x)=\{y \in Y: g(x, y) \geq t\} \text { for all } x \in X \text {. }
$$


Let

$$
\begin{aligned}
& M=\{(x, y) \in X \times Y: e(x, y) \geq t\}, \\
& N=\{(x, y) \in X \times Y: f(x, y) \geq t\}, \quad \text { and } \\
& P=\{(x, y) \in X \times Y: y \in T x\} .
\end{aligned}
$$

It is easy to check that $M, N$ and $C$ satisfy conditions (i)-(iii) of Theorem 2.1. By (3.4), $P \subset M$, and by Proposition 7 in [1, p. 110], $P$ is closed in $X \times Y$. For each $x \in X$, the set

$$
\{y \in K:(x, y) \in P\}=\bigcup_{x \in X} S(x) \cap T x
$$

is nonempty acyclic. So $K$ and $P$ satisfy condition (iv) of Theorem 2.1. Hence by Theorem 2.1,

$$
\bigcap_{x \in X} C(x) \cap K \neq \emptyset
$$

that is, there exists $y_{0} \in K$ such that $g\left(x, y_{0}\right) \geq t$ for all $x \in X$. Therefore we have

$$
\sup _{y \in Y} \inf _{x \in X} g(x, y) \geq t .
$$

This contradicts the choice of $t$. Therefore (3.3) is proved. This completes the proof.

Remark 3.3. Theorem 3.2 generalizes Theorem 2.1 in [17] from topological vector spaces to general topological spaces without convexity structure. Furthermore, Theorem 3.2 differs from Theorem 2.1 in [17] on the method of proof.

Corollary 3.3. Let $X, Y, S, T$, and e, $f, g$ be as in Theorem 3.2. Assume further, that given $\lambda \in \mathbb{R}$, we have

$$
\inf _{x \in X, y \in T x} e(x, y) \geq \lambda .
$$

Then there exists $y_{0} \in Y$ such that $g\left(x, y_{0}\right) \geq \lambda$ for all $x \in X$.

Remark 3.4. Corollary 3.3 generalizes Corollary 2.4 in [10] from $H$-spaces to general topological spaces without convexity structure.

The following two minimax inequalities are obtained from Theorem 3.2 as special cases by taking $f=g$ and $e=f=g$, respectively. 
Theorem 3.3. Let $X, Y, S$, and $T$ be as in Theorem 3.2. Let e, $f: X \times$ $Y \rightarrow \mathbb{R}$ be functions and

$$
\beta=\sup _{y \in Y} \inf _{x \in X} f(x, y) .
$$

Suppose the following conditions are fulfilled:

(i) for each $t>\beta, f(x, y)$ is t-transfer compactly upper semicontinuous in $y$;

(ii) for each $A \in\langle X\rangle$ with

$$
A \cap\{x \in X: f(x, y)<t\} \neq \emptyset
$$

for each $t>\beta$ and $y \in Y$, there exists a continuous mapping $\varphi_{A}: \Delta_{|A|-1} \rightarrow X$ such that

$$
\varphi_{A}\left(\Delta_{|A \cap\{x \in X: f(x, y)<t\}|-1}\right) \subset\{x \in X: e(x, y)<t\}
$$

for all $t>\beta$ and $y \in Y$.

Then

$$
\inf _{x \in X, y \in T x} e(x, y) \leq \sup _{y \in Y} \inf _{x \in X} f(x, y)
$$

Theorem 3.4. Let $X, Y, S$, and $T$ be as in Theorem 3.2. Let $f: X \times Y \rightarrow$ $\mathbb{R}$ be a function and

$$
\beta=\sup _{y \in Y} \inf _{x \in X} f(x, y) .
$$

Suppose the following conditions are fulfilled:

(i) for each $t>\beta, f(x, y)$ is t-transfer compactly upper semicontinuous in $y$;

(ii) for each $A \in\langle X\rangle$ with

$$
A \cap\{x \in X: f(x, y)<t\} \neq \emptyset
$$

for each $t>\beta$ and $y \in Y$, there exists a continuous mapping $\varphi_{A}: \Delta_{|A|-1} \rightarrow X$ such that

$$
\varphi_{A}\left(\Delta_{|A \cap\{x \in X: f(x, y)<t\}|-1}\right) \subset\{x \in X: f(x, y)<t\}
$$

for all $t>\beta$ and $y \in Y$.

Then

$$
\inf _{x \in X, y \in T x} f(x, y) \leq \sup _{y \in Y} \inf _{x \in X} f(x, y) .
$$

Remark 3.5. Theorem 3.4 generalizes Theorem 1 in [8] in several aspects.

By using Theorems 3.2 and 3.4, we can obtain the following Theorems 3.5 and 3.6, which are the generalizations of Fan's minimax inequality. 
Theorem 3.5. Let $Y$ be a topological space, $X \subset K, K \subset Y$ be nonempty subsets and $K$ is compact. Let $e, f, g: X \times Y \rightarrow \mathbb{R}$ be functions satisfying conditions (i)-(iii) of Theorem 3.2. Then

$$
\inf _{x \in X} e(x, x) \leq \sup _{y \in Y} \inf _{x \in X} g(x, y) .
$$

Proof. Define the set-valued mapping $S: X \rightarrow 2^{Y}$ by $S(x)=K$ for each $x \in X$. The set-valued mapping $T$ is defined by $T(x)=\{x\}$ for each $x \in X$, then the conclusion follows from Theorem 3.2. This completes the proof.

Theorem 3.6. Let $X, Y, K$ be as in Theorem 3.5. Let $f: X \times Y \rightarrow \mathbb{R}$ be a function satisfying conditions (i)-(ii) of Theorem 3.4. Then

$$
\inf _{x \in X} f(x, x) \leq \sup _{y \in X} \inf _{x \in X} f(x, y) .
$$

Proof. We define the set-valued mappings $S, T: X \rightarrow 2^{Y}$ by $S(x)=K$ and $T(x)=\{x\}$ for each $x \in X$, respectively, then the result follows from Theorem 3.4. This completes the proof.

Remark 3.6. In Theorems 3.5 and 3.6, $X$ or $Y$ need not to be compact, thus, Theorems 3.5 and 3.6 are the generalizations of Theorems 2.5 and 2.6 in [10] and Theorem 5 in [5].

As another application of Theorem 3.2, we have the following theorem.

Theorem 3.7. Let $X, Y, S$, and $T$ be as in Theorem 3.2. Let e, $f, g: X \times$ $Y \rightarrow \mathbb{R}$ be functions and

$$
\beta=\inf _{y \in Y} \sup _{x \in X} f(x, y)>-\infty .
$$

Suppose the following conditions are fulfilled:

(i) for each $t>-\beta, f(x, y)$ is -t-transfer compactly lower semicontinuous in $y$;

(ii) for each $(x, y) \in X \times Y, f(x, y) \leq g(x, y)$;

(iii) for each $A \in\langle X\rangle$ with

$$
A \cap\{x \in X: g(x, y)>-t\} \neq \emptyset
$$

for all $t>-\beta$ and $y \in Y$, there exists a continuous mapping $\varphi_{A}: \Delta_{|A|-1} \rightarrow X$ such that

$$
\varphi_{A}\left(\Delta_{|A \cap\{x \in X: g(x, y)>-t\}|-1}\right) \subset\{x \in X: e(x, y)>-t\}
$$

for each $t>-\beta$ and $y \in Y$. 
Then

$$
\inf _{y \in Y} \sup _{x \in X} f(x, y) \leq \sup _{x \in X, y \in T x} e(x, y)
$$

Proof. Set $e^{\prime}=-e, f^{\prime}=-g$, and $g^{\prime}=-f$, then $e^{\prime}, f^{\prime}$, and $g^{\prime}$ satisfy conditions (i)-(iii) of Theorem 3.2. Thus, by Theorem 3.2,

$$
\inf _{x \in X, y \in T x} e^{\prime}(x, y) \leq \sup _{y \in Y} \inf _{x \in X} g^{\prime}(x, y) \text {, }
$$

that is,

$$
\inf _{y \in Y} \sup _{x \in X} f(x, y) \leq \sup _{x \in X, y \in T x} e(x, y) .
$$

This completes the proof.

By taking $f=g$, and then $T(x)=\{x\}$ for each $x \in X$ in Theorem 3.7, we get the following minimax inequalities. We omit the proofs.

Theorem 3.8. Let $X, Y, S$, and $T$ be as in Theorem 3.2. Let $e, f: X \times$ $Y \rightarrow \mathbb{R}$ be functions and

$$
\beta=\inf _{y \in Y} \sup _{x \in X} f(x, y)>-\infty
$$

such that

(i) for each $t>-\beta, f(x, y)$ is -t-transfer compactly lower semicontinuous in $y$;

(ii) for each $A \in\langle X\rangle$ with

$$
A \cap\{x \in X: f(x, y)>-t\} \neq \emptyset
$$

for all $t>-\beta$ and $y \in Y$, there exists a continuous mapping $\varphi_{A}: \Delta_{|A|-1} \rightarrow X$ such that

$$
\varphi_{A}\left(\Delta_{|A \cap\{x \in X: f(x, y)>-t\}|-1)}\right)\{x \in X: e(x, y)>-t\}
$$

for each $t>-\beta$ and $y \in Y$.

Then

$$
\inf _{y \in Y} \sup _{x \in X} f(x, y) \leq \sup _{x \in X, y \in T x} e(x, y)
$$

Remark 3.7. Theorem 3.8 is different from Theorem 3 in [14] for mappings with noncompact domains and $Y$ need not be locally convex and complete.

Theorem 3.9. Let $Y$ be a topological space, $X \subset K, K \subset Y$ be nonempty subsets and $K$ is compact. Let $e, f, g: X \times Y \rightarrow \mathbb{R}$ be functions satisfying conditions (i)-(iii) of Theorem 3.7. Then

$$
\inf _{y \in Y} \sup _{x \in X} f(x, y) \leq \sup _{x \in X} e(x, x) .
$$


Theorem 3.10. Let $X, Y, K$ be as in Theorem 3.9. Let e, $f: X \times Y \rightarrow \mathbb{R}$ be functions satisfying conditions (i)-(ii) of Theorem 3.8. Then

$$
\inf _{y \in Y} \sup _{x \in X} f(x, y) \leq \sup _{x \in X} e(x, x) .
$$

Remark 3.8. In Theorems 3.9 and $3.10, X$ or $Y$ need not to be compact, thus, Theorems 3.9 and 3.10 are new minimax inequalities of Ky Fan type.

Acknowledgement. The author wishes to express his gratitude to the referees for their suggestions.

\section{References}

[1] Aubin, J.P., Ekeland, I., Applied Nonlinear Analysis, John Wiley \& Sons, New York, 1984.

[2] Bardaro, C., Ceppitelli, R., Some further generalizations of Knaster-KuratowskiMazurkiewics theorem and minimax inequalities, J. Math. Anal. Appl. 132(3) (1988), 484-490.

[3] Ding, X. P., Generalized $G-K K M$ theorems in generalized convex spaces and their applications, J. Math. Anal. Appl. 266 (2002), 21-37.

[4] Fan, K., A generalization of Tychonoff's fixed point theorem, Math. Ann. 142 (1961), 305-310.

[5] Fan, K., Some properties of convex sets relation to fixed point theorems, Math. Ann. 266 (1984), 519-537.

[6] Granas, A., Liu, F. C., Coincidence for set-valued maps and minimax inequalities, J. Math. Pures Appl. (9) 65 (1984), 119-148.

[7] Ha, C. W., Minimax and fixed point theorems, Math. Ann. 248 (1980), 73-77.

[8] Ha, C. W., On a minimax inequality of Ky Fan, Proc. Amer. Math. Soc. 99 (1987), 680-682.

[9] Lu, H. S., Tang, D. S., An intersection theorem in L-convex spaces with applications, J. Math. Anal. Appl. 312 (2005), 343-356.

[10] Lu, H. S., Zhang, J. H., Minimax inequalities in the spaces without linear structure, Taiwanese J. Math. 8(4) (2004), 703-712.

[11] McClendon, J. F., Minimax and variational inequalities for compact spaces, Proc. Amer. Math. Soc. 89(4) (1983), 717-721.

[12] Shioji, N., A further generalization of Knaster-Kuratowski-Mazurkiewics theorem, Proc. Amer. Math. Soc. 117 (1991), 187-195.

[13] Tarafdar, E., Fixed-point theorems in H-spaces and equilibrium point of abstract economies, J. Austral. Math. Soc. Ser. A 53 (1992), 252-260.

[14] Tarafdar, E., Watson, P. J., A coincidence point theorem and related results, Appl. Math. Lett. 11(1) (1998), 37-40.

[15] Tian, G. Q., Generalization of FKKM theorem and the Ky Fan minimax inequality with applications to maximal elements, price equilibrium and complementarity, J. Math. Anal. Appl. 170 (1992), 457-471.

[16] Zhang, J .H., Ma, R. Y., Minimax inequalities of Ky Fan, Appl. Math. Lett. 11 (1998), 37-41. 
[17] Zhang, J. H., Some minimax inequalities for mappings with noncompact domain, Appl. Math. Lett. 17 (2004), 717-720.

H.-S. LU

SCHOOL OF ECONOMICS AND MANAGEMENT

JiAngSU TEAChers University OF TEChNOLOGY

Changzhou 213001

People's Republic of China

E-MAIL: HAISHULU@SINA.COM 